\title{
Technique of video-assisted thoracoscopic left pneumonectomy
}

\author{
Mark W. Hennon ${ }^{1,2}$, Todd L. Demmy ${ }^{1,2}$ \\ ${ }^{1}$ Department of Thoracic Surgery, Roswell Park Cancer Institute, Buffalo, NY, USA; ${ }^{2}$ Department of Surgery, Jacobs School of Medicine and \\ Biomedical Sciences, Buffalo, NY, USA \\ Correspondence to: Mark W. Hennon, MD, FACS. Department of Thoracic Surgery, Roswell Park Cancer Institute, Elm and Carlton Streets, Buffalo, \\ NY 14263, USA. Email: mark.hennon@roswellpark.org; Todd L. Demmy, MD, FACS. Professor of Oncology, Department of Thoracic Surgery, \\ Roswell Park Cancer Institute, Elm and Carlton Streets, Buffalo, NY 14263, USA. Email: todd.demmy@roswellpark.org.
}

\begin{abstract}
Video-assisted thoracoscopic surgery (VATS) approaches to lobectomy for treatment of early stage non-small cell lung cancer (NSCLC) have generally been accepted to be beneficial. Experience and results for more extensive resections, including thoracoscopic pneumonectomy are limited. Here we report a case with attached videos describing key technical aspects of performing a thoracoscopic left pneumonectomy. This demonstrates the adoption of VATS for tumor pathology requiring pneumonectomy is feasible and can be done safely. Further study is needed to clarify potential advantages or drawbacks to approaching more complex tumor pathology by VATS.
\end{abstract}

Keywords: Video-assisted thoracoscopic surgery (VATS); left pneumonectomy; thoracoscopic

Received: 22 December 2016; Accepted: 06 January 2017; Published: 17 March 2017.

doi: 10.21037 /jovs.2017.02.06

View this article at: http://dx.doi.org/10.21037/jovs.2017.02.06

\section{Introduction}

Since the first reported video-assisted thoracoscopic surgery (VATS) lobectomy by Walker in April 1992, evidence has accumulated documenting the advantages of thoracoscopic approaches over thoracotomies for early stage non-small cell lung cancer (NSCLC) anatomic lung resections (1-6). It is not known whether these benefits translate to patients with more complex tumor pathologies. Initial concerns regarding the oncologic validity of VATS for early stage disease have largely been disproven, leading one to suppose that more complex tumor pathology is approachable provided open oncologic principles are maintained.

Despite these advantages, widespread adoption of a VATS approach to lobectomy progressed at a relatively slow pace. Experience and results for thoracoscopic pneumonectomy are even more limited since the first report by Walker in 1994 (7). Many of the hurdles that contributed to the slow adoption of VATS approaches for lobectomy exist for performing a total lung resection. Given the potential for faster initiation of adjuvant therapy or recovery after induction therapies in patients undergoing thoracoscopic approaches, VATS offers promising approaches for patients whose longevities are often compromised by occult systemic metastases (8).
With those principles in mind, surgeons are adopting minimally invasive approaches to lung resections previously deemed unsuitable for thoracoscopy. Surgical instrumentation and video exposure capabilities continue to evolve, and it is now possible to replicate most open surgical techniques minimally invasively as many thoracic surgeons are using VATS or robotic techniques as their primary approaches. Thus, it is reasonable to challenge previously reported exclusion criteria for a minimally invasive approaches (9). Reports are accumulating demonstrating the feasibility and safety of VATS for complex resections, such as en bloc chest wall, lobar bronchoplasty/bronchial sleeve, and VATS pneumonectomy (10-13).

\section{Case presentation}

The patient was an 80-year-old man who presented with a cough and hemoptysis with what felt like "cold symptoms". He had a 60 pack year smoking history. His past medical history was significant for gastroesophageal reflux disease, hyperlipidemia, hypertension, prostate cancer (stage 2), sleep apnea, hearing loss, and cataracts. Diagnostic bronchoscopy with biopsies revealed non-small cell lung carcinoma (large 


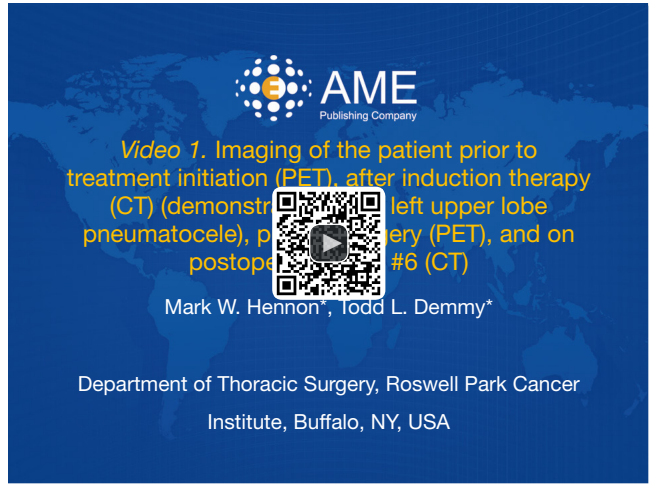

Figure 1 Imaging of the patient prior to treatment initiation (PET), after induction therapy (CT) (demonstrating large left upper lobe pneumatocele), prior to surgery (PET), and on postoperative day \#6 (CT) (14). Available online: http://www.asvide.com/articles/1412

cell neuroendocrine variant). Computed tomography demonstrated a $7.4 \mathrm{~cm}$ left upper lobe mass with hilar lymphadenopathy abutting the main pulmonary artery and extending into the lower lobe (Figure 1). PET/CT did not reveal any evidence of metastatic disease (tumor SUV $=31$ ) (Figure 1). He underwent neoadjuvant chemotherapy and radiation with a nearly complete response but he developed a sizable pneumatocele with production of large quantities of foul sputum associated with significant deconditioning and bedridden performance status requiring hospitalization with discharge to a rehabilitation facility. On ambulatory preoperative evaluation, he was noted to be very frail and was referred for preoperative physical therapy for progressive strength training. After one month, the patient returned in better condition with a Zubrod performance of 1 . Pulmonary function testing (PFTs) showed $\mathrm{FEV}_{1}$ to be $73 \%$ predicted and DLCO $52 \%$ predicted. Lung perfusion scanning showed only $17 \%$ of total pulmonary function on the left.

\section{Preoperative preparation}

The patient's preoperative evaluation for a possible thoracoscopic left pneumonectomy did not differ from that for open pneumonectomy. A standard cardiopulmonary work up was performed including routine transthoracic echocardiogram and PFTs. Split lung perfusion testing and cardiopulmonary exercise testing are obtained in marginal candidates. An additional option (not required for this patient) is intraoperative transesophageal echocardiography (TEE) to assess cardiac function at the time of pulmonary artery clamping. When there are conflicting or borderline predictive data, useful information can be obtained preoperatively by right heart catheterization. This is done by ipsilateral pulmonary artery balloon-occlusion while pharmacologically increasing the cardiac output, measuring right ventricular pressure response, and assessing systemic arterial oxygen saturations. Given the high morbidity and mortality rates associated with pneumonectomy, sleeve resection (open or thoracoscopic) is performed, whenever possible, to spare lung function.

\section{Equipment preference card}

* Flexible intubating bronchoscope;

* Mediastinoscopy scope and tools;

* Low profile 5-mm round shafted thoracoscopic angled graspers (Sontec Instruments, Inc., Centennial, CO or Dufner Instruments, Tuttlingen, Germany);

* Alexis wound retractor, size small (Rancho Santa Margarita, CA, USA);

* Flexible-tip video laparoscope, $5 \mathrm{~mm}$ or $10 \mathrm{~mm}$ (Olympus, Center Valley, PA, USA);

* Harken \#1 and \#2, Rochester Pean, and HarringtonMixter surgical clamps;

* Thoracoscopic needle driver;

* Chitwood knot pusher (Scanlon International, Inc., St. Paul, MN, USA);

* Thoracoscopic DeBakey Forceps (Heartport, Inc. or Sontec instruments, Inc.);

* Endoscopic sheers;

* 5-mm thoracoscopic suction (Sontec Instruments, Inc., Centennial, CO, USA);

* Endo Kittner Dissector;

* Long scalpel handle with 15 blade;

* Endoscopic surgical stapler, with vascular reloads, and thick tissue reloads $(4.8 \mathrm{~mm})$;

* Ligasure sealing device (Covidien-Medtronic, Minneapolis, MN, USA);

* Aquamantys bipolar energy devices, particularly MBS and VATS sealers, (Medtronic, Minneapolis, MN, USA);

* Red rubber catheter(s), latex and latex-free;

* Laparoscopic flexible liver retractor $(5 \mathrm{~mm})$ such as the Diamond-Flex ${ }^{\mathrm{TM}}$ (Carefusion, San Diego, CA, USA);

* Anchor tissue retrieval bag (Anchor Products Co., IL, USA);

* Balanced drainage system, Pleur-evac ${ }^{\circledR}$ Pneumonectomy Unit (Teleflex ${ }^{\circledR}$, Research Triangle Park, NC, USA).

\section{Procedure}

The patient underwent mediastinoscopy for staging and 


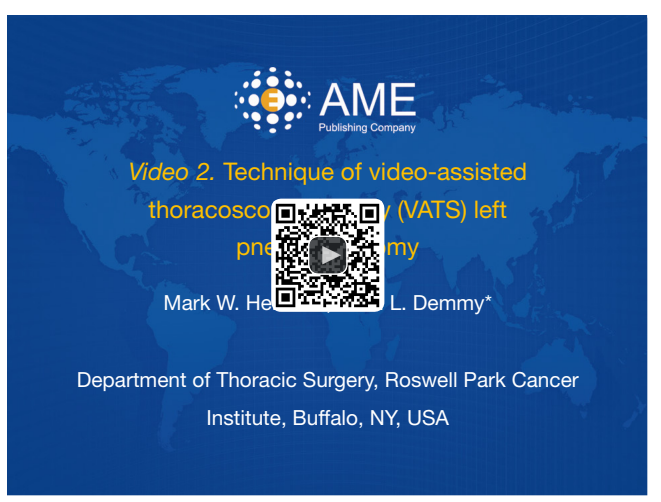

Figure 2 Technique of video-assisted thoracoscopic surgery (VATS) left pneumonectomy (15).

Available online: http://www.asvide.com/articles/1413

airway mobilization, left VATS with extensive lysis of adhesions caused by the necrotic lung, left pneumonectomy, resection of sympathetic chain and portion of the vagus nerve, coverage of the bronchial stump with pericardial fat pad, and mediastinal lymph node dissection (Figure 2). Preoperatively, VATS left upper lobectomy was a consideration, but intraoperative findings showed extensive induration and inflammation extending along the posterior fissure and into the main pulmonary artery and lower lobe. The area of previous tumor involvement could not be entirely resected without pneumonectomy; in fact the area of treatment effect with scattered residual tumor cells measured over $10 \mathrm{~cm}$ on final pathologic review.

The patient was positioned in right lateral decubitus position with the table flexed. Careful padding is needed in large or frail patients to prevent pressure injuries. Standard VATS lobectomy incisions $(1-2 \mathrm{~cm})$ are created in the 8 th intercostal space in the posterior axillary line, the sixth intercostal space more anteriorly, and a $4 \mathrm{~cm}$ access incision in the anterior $4^{\text {th }}$ intercostal space (the $3^{\text {rd }}$ and $4^{\text {th }}$ space are palpated or visualized internally to choose a wide interspace to enable dissection and later extraction of the specimen).

Important anatomic considerations for this VATS left pneumonectomy involved isolating both the inferior and superior pulmonary veins prior to dividing either one of them to minimize vascular congestion that may occur in the lung while time is spent isolating and dividing the pulmonary artery. While such congestion may not be problem in every case, even a mild amount of engorgement can make the specimen more difficult to extract, increase parenchymal oozing, and impair exposure because of crowding the working space. Often the effects of tumor or induction therapy will require the vein dissection to be carried out within the pericardial cavity as demonstrated in this case. By dissecting both veins and dividing them in rapid succession, attention could be turned towards isolating the main left pulmonary artery.

To isolate the left main pulmonary artery the lung was retracted superiorly. Blunt dissection was performed paying careful attention to dissect against the left mainstem bronchus (creating a space between the pulmonary artery and airway). When this was completed, a red rubber catheter was positioned in between these two major structures. This catheter was then swung around the pulmonary artery (reverse exclusion technique) instead of the bronchus. Using this catheter as a gentle traction device around the pulmonary artery, remaining tissues (lymphatics, vagus nerve, thickened pleura, and residual pericardium) were dissected away. Thickened tissue or other dissection devices like metallic clips can potentially interfere with stapler passage or function. The catheter then serves as a leader to safely pass a vascular load of an endoscopic stapler across the left main pulmonary artery. We prefer exchanging the soft latex catheter with a latex free (stiffer) plastic because this reliably guides the anvil around thick tissues. Also, advanced tumors have exposure limitations that often require changing camera vantage points to dissect safely. Frequently multiple $5 \mathrm{~mm}$ retractors are needed to optimize the exposures and dissection instruments and retractors can easily share ports with a $5 \mathrm{~mm}$ camera.

Prior to dividing the artery, the stapler was closed to assess for any hemodynamic compromise that may result from distorting the heart, main or contralateral pulmonary artery. It is important to use a stapler system that can close gently enough during test clamping not to crush and disrupt the delicate pulmonary artery. While we have never had a catastrophic failure dividing the main pulmonary artery with staplers, this is concern for many surgeons who may prefer to use a product that seals but does not divide the artery (e.g., Serial TA stapling or a "no-knife" instrument). The artery can then be cut between staple lines that have been inspected.

Once the pulmonary artery was divided, the bronchus was dissected up to the level of the carina to avoid a long bronchial stump just as with an open resection. This was aided greatly by using a $5 \mathrm{~mm}$ laparoscopic flexible liver retractor such as the Diamond-Flex ${ }^{\mathrm{TM}}$ (Carefusion, San Diego, CA, USA). The stapler was placed through the posterior inferior port site with an anterior $5 \mathrm{~mm}$ camera position sharing the port with the Diamond-Flex ${ }^{\mathrm{TM}}$ retractor. The endoscopic stapler can also be placed next to the laparoscopic retractor if a better angle is 
needed for division. Alternatively, a standard open articulating TA 45 stapler just fits through the access incision but an angled knife is needed to amputate the stump after firing. This technique can also be used after the main specimen is removed if the surgeon needs to divide the stump closer to the carina in the event of a positive margin or excessive length. Pericardial fat was then mobilized and anchored over the left bronchial stump. We have also harvested intercostal muscle, as necessary, for stump coverage.

\section{Role of team members}

The patient's operation was performed in an academic institution by an attending surgeon with the assistance of a surgical trainee.

\section{Postoperative management}

Postoperative care is essentially the same for VATS left pneumonectomy as open cases. Close attention to fluid status is of critical importance, and balanced drain chest tube drainage with the Pleur-evac ${ }^{\circledR}$ Pneumonectomy Unit (Teleflex ${ }^{\circledR}$, Research Triangle Park, NC) is employed until the morning of POD\#1.

This patient did well postoperatively, experiencing only minor complications of exacerbation of pre-existing tachycardia (controlled with amiodarone) and hoarseness, despite intact vocal cord function. He was discharged on the eighth post-operative day to rehabilitation, which was planned preoperatively based on his preoperative physical therapy needs and he was discharged home from rehab 6 days later presenting to clinic without the need for opioid analgesics, a stable Horner's syndrome from the apical extrapleural dissection, and no symptoms except mild dyspnea on exertion. Final pathology showed no nodal metastases and no residual disease other than small clusters of malignant cells in the previous tumor bed and irradiated lung tissue. No adjuvant therapy is planned.

\section{Tips, tricks, and pitfalls}

Dissecting both pulmonary veins and isolating them with vessel loops is critical so that they can be divided in succession without delay, minimizing vascular congestion of the lung that can occur while dissecting the pulmonary artery. Performing the dissection from within the pericardium is often necessary as a result of tumor or treatment effects.

Retraction of the whole lung (which can be difficult) superiorly with a $5 \mathrm{~mm}$ laparoscopic flexible liver retractor such as the Diamond-Flex ${ }^{\mathrm{TM}}$ (Carefusion, San Diego, CA, USA) greatly facilitates dissection between the pulmonary artery and bronchus. We sometimes apply this retractor after division of the veins (to assist with the dissection of the pulmonary artery) or just to facilitate dissection and elongation of the bronchus as in this video.

Using the red rubber catheter as a gentle traction device around the PA, allows for safe dissection of any residual tissue on the artery like attached lymphatics or residual pericardium that could interfere with stapler function. It is then used as a leader to safely pass a vascular load of an endoscopic stapler across the left main pulmonary artery.

We do not advocate passing the stapler across the main pulmonary artery without the red rubber catheter serving as a leader because it reduces the opportunity for the stapler anvil to create an injury. We also believe that dividing the bronchus before the artery is risky because releasing the airway tether concentrates retraction force on the blood vessel which is delicate and can rupture catastrophically.

\section{Discussion}

Our approach to thoracoscopic left pneumonectomy for an elderly patient with a central non-small cell lung carcinoma without evidence of metastatic disease is described in detail. The patient underwent preoperative chemotherapy and radiation and given his age, deconditioning, and medical comorbidities was felt to be too high risk for thoracotomy. Due to limited experience, large series examining results for thoracoscopic resection for locally advanced, more complex tumors are lacking. Therefore, existing evidence does not clearly define advantages for a thoracoscopic approach to these resections. Challenges facing surgeons performing minimally invasive pneumonectomy mirror those that existed for VATS lobectomy in its infancy. However, loss of vascular control should a proximal vessel injury occurs is the main concern to most physicians. Specimen extraction can be difficult given the large size comparable to small VATS incisions. The central nature of some tumors and the effects of frequently applied chemoradiation can also lead to difficult hilar dissection.

A retrospective review of all patients undergoing pneumonectomy from $1 / 2002$ to $12 / 2012$ at our institution included 107 consecutive cases. Forty were approached via standard thoracotomy, while 67 pneumonectomies were attempted by VATS. In 17 cases, conversion from VATS to thoracotomy was required. Preoperative characteristics were similar in the groups except for greater age, female sex, 
and preoperative comorbidities in the VATS group. Not surprisingly, a learning curve was apparent as the percentage of successful completion of VATS pneumonectomy rose from $50 \%$ to $82 \%$ during the second half of the series. There were no intraoperative deaths from bleeding or other technical mishaps. In all cases the pulmonary artery was able to be controlled safely. Using intent to treat analysis, perioperative outcomes and survival curves matched by stages were similar (16).

In summary, approaching pneumonectomy by VATS appears to be a safe strategy that does not compromise oncologic principles. When approaching more extensive resections such as pneumonectomy or bronchial sleeve resection with or without arterioplasty, the importance of gaining proximal control of the main pulmonary artery cannot be overstated. The timing of this maneuver occurs after dissection and division of both pulmonary veins. Obtaining control of the main pulmonary artery is the most critical and stress-inducing step and can be facilitated by maneuvers like intrapericardial dissection, initial dissection of the bronchus away from the artery, catheter sling maneuvers, and mobilization of related structures such as the airway by cervical mediastinoscopy.

\section{Acknowledgements}

None.

\section{Footnote}

Conflict of Interest: The authors have no conflicts of interest to declare.

Informed Consent: Written informed consent was obtained from the patient for publication of this manuscript and any accompanying images.

\section{References}

1. Walker WS, Carnochan FM, Tin M. Thoracoscopy assisted pulmonary lobectomy. Thorax 1993;48:921-4.

2. Hartwig MG, D'Amico TA. Thoracoscopic lobectomy: the gold standard for early-stage lung cancer? Ann Thorac Surg 2010;89:S2098-101.

3. Paul S, Altorki NK, Sheng S, et al. Thoracoscopic lobectomy is associated with lower morbidity than open lobectomy: a propensity-matched analysis from the STS database. J Thorac Cardiovasc Surg 2010;139:366-78.

4. McKenna RJ Jr, Houck W, Fuller CB. Video-assisted thoracic surgery lobectomy: experience with 1,100 cases. Ann Thorac Surg 2006;81:421-5; discussion 425-6.

5. Villamizar NR, Darrabie MD, Burfeind WR, et al. Thoracoscopic lobectomy is associated with lower morbidity compared with thoracotomy. J Thorac Cardiovasc Surg 2009;138:419-25.

6. Nwogu CE, D'Cunha J, Pang H, et al. VATS lobectomy has better perioperative outcomes than open lobectomy: CALGB 31001, an ancillary analysis of CALGB 140202 (Alliance). Ann Thorac Surg 2015;99:399-405.

7. Walker WS, Carnochan FM, Mattar S. Video-assisted thoracoscopic pneumonectomy. Br J Surg 1994;81:81-2.

8. Petersen RP, Pham D, Burfeind WR, et al. Thoracoscopic lobectomy facilitates the delivery of chemotherapy after resection for lung cancer. Ann Thorac Surg 2007;83:12459; discussion 1250.

9. Hanna JM, Berry MF, D'Amico TA. Contraindications of video-assisted thoracoscopic surgical lobectomy and determinants of conversion to open. J Thorac Dis 2013;5 Suppl 3:S182-9.

10. Nakagiri T, Akashi A, Shigemura N. Thoracoscopic rib resection using a Gigli saw. Ann Thorac Surg 2005;80:755-6.

11. Rocco G, Fazioli F, Martucci N, et al. Video-assisted thoracic surgery rib resection and reconstruction with titanium plate. Ann Thorac Surg 2011;92:744-5.

12. Sahai RK, Nwogu CE, Yendamuri S, et al. Is thoracoscopic pneumonectomy safe? Ann Thorac Surg 2009;88:1086-92.

13. Nwogu CE, Glinianski M, Demmy TL. Minimally invasive pneumonectomy. Ann Thorac Surg 2006;82:e3-4.

14. Hennon MW, Demmy TL. Imaging of the patient prior to treatment initiation (PET), after induction therapy (CT) (demonstrating large left upper lobe pneumatocele), prior to surgery (PET), and on postoperative day \#6 (CT). Asvide 2017;4:104. Available online: http://www.asvide. com/articles/1412

15. Hennon MW, Demmy TL. Technique of video-assisted thoracoscopic surgery (VATS) left pneumonectomy. Asvide 2017;4:105. Available online: http://www.asvide.com/ articles $/ 1413$

16. Battoo A, Jahan A, Yang Z, et al. Thoracoscopic pneumonectomy: an 11-year experience. Chest 2014;146:1300-9.

doi: $10.21037 /$ jovs.2017.02.06

Cite this article as: Hennon MW, Demmy TL. Technique of video-assisted thoracoscopic left pneumonectomy. J Vis Surg 2017;3:32. 